CARDIOVASCULAR MEDICINE

\title{
Effects of magnesium on atrial fibrillation after cardiac surgery: a meta-analysis
}

\author{
S Miller, E Crystal, M Garfinkle, C Lau, I Lashevsky, S J Connolly
}

Heart 2005;91:618-623. doi: 10.1136/hrt.2004.033811

See end of article for authors' affiliations

Correspondence to:

Dr Eugene Crystal,

Electrophysiology

Program, Schulich Heart

Centre, Sunnybrook and

Women's College Health

Sciences Centre, 2075

Bayview Avenue, Toronto,

Ontario, Canada M4N

3M5; eugene.crystal@

sw.ca

Accepted 7 June 2004
Objectives: To assess the efficacy of the administration of magnesium as a method for the prevention of postoperative atrial fibrillation (AF) and to evaluate its influence on hospital length of stay (LOS) and mortality.

Methods: Literature search and meta-analysis of the randomised control studies published since 1966.

Results: 20 randomised trials were identified, enrolling a total of 2490 patients. Study sample size varied between 20 and 400 patients. Magnesium administration decreased the proportion of patients developing postoperative AF from $28 \%$ in the control group to $18 \%$ in the treatment group lodds ratio $0.54,95 \%$ confidence interval (Cl) 0.38 to 0.75 ). Data on LOS were available from seven trials (1227 patients). Magnesium did not significantly affect LOS (weighted mean difference -0.07 days of stay, $95 \% \mathrm{Cl}-0.66$ to 0.53$)$. The overall mortality was low $(0.7 \%)$ and was not affected by magnesium administration (odds ratio $1.22,95 \% \mathrm{Cl} 0.39$ to 3.77 ).

Conclusion: Magnesium administration is an effective prophylactic measure for the prevention of postoperative AF. It does not significantly alter LOS or in-hospital mortality.
A trial fibrillation $(\mathrm{AF})$ is a common complication after cardiac surgery. Most studies suggest that the frequency ranges between $25-40 \% .^{1-3}$ Several reports have indicated that postoperative $\mathrm{AF}$ is associated with an increased length of stay (LOS) in hospital and consequently a greater utilisation of health care resources. ${ }^{45}$ Postoperative AF is also associated with higher rates of postoperative stroke, compromised cardiac function, and adverse effects from drugs used to prevent $\mathrm{AF}^{6}{ }^{6}$ Despite many years of clinical experience and a large amount of investigation, prevention and treatment of postoperative $\mathrm{AF}$ remain controversial. Many questions about the mechanisms and pathophysiology of AF remain unanswered, ${ }^{4}$ further contributing to the ambiguity in reaching consensus about appropriate treatment. Increasing patient age is generally considered the greatest risk factor for postoperative AF and an aging population suggests that postoperative AF will continue to be a considerable problem in the future. ${ }^{8}$

Some studies have shown that serum hypomagnesaemia is common after coronary artery bypass grafts (CABG) and other types of cardiac surgery ${ }^{9-12}$ and is associated with postoperative morbidity such as atrial tachyarrhythmia. ${ }^{10}$ However, this has not been confirmed by all research. ${ }^{13}$

Some clinical trials have assessed the efficacy of magnesium as a method of intervention to reduce the incidence of postoperative AF. Often these studies were statistically underpowered and the results have been inconsistent.

We undertook a systematic review of the evidence from randomised controlled trials to assess the effectiveness of magnesium as a method for prevention of postoperative AF and to evaluate its influence on the incidence of postoperative stroke, mortality, and LOS.

\section{METHODS}

\section{Search strategy}

Titles and abstracts (where available) from Medline/PubMed and the Cochrane Central Register of Controlled Trials (CENTRAL) library database were searched from 1966 to July 2003. The search terms were "atrial fibrillation", "atrial flutter", "surgery", and "magnesium". No language restrictions were applied. All references from papers obtained through the databases were searched by hand. References from papers obtained through the above procedure were searched for additional studies to be included.

Abstracts from the annual scientific meetings of the American College of Cardiology, American Heart Association, British Cardiac Society (Heart), European Society of Cardiology, and North American Society for Pacing and Electrophysiology between 2000 and 2003 were searched.

\section{Inclusion criteria for studies}

Studies were included only if they met all of the following criteria:

- Randomised allocation of patients;

- placebo used as the control treatment

- well described protocol of magnesium administration as the intervention

- adequate data on treatment efficacy (supraventricular arrhythmia incidence)

- primary prevention of postoperative AF in CABG or valve surgery

- treatment started immediately preoperatively, perioperatively, or postoperatively;

- no confounding randomised drug treatment.

Double blind and non-blinded studies were included. Two investigators (EC, SM) evaluated the design of each trial for inclusion.

The primary outcome measure was the incidence of postoperative AF or atrial flutter except where total incidence of supraventricular arrhythmia was documented. Two other outcome measures, LOS and incidence of stroke, were also analysed when they could be obtained from the publication.

Abbreviations: $A F$, atrial fibrillation; $C A B G$, coronary artery bypass graft; CENTRAL, Cochrane Central Register of Controlled Trials; Cl, confidence interval; LOS, length of stay; OR, odds ratio 
When the allocation of patients was not clear, the authors were approached. Two authors were contacted regarding the division of patients into control and treatment groups ${ }^{14}{ }^{15}$ but did not respond.

\section{Data collection and analysis}

Two reviewers (MG, SM) independently extracted data into a collection table prepared for the study. All discrepancies between individually collected data were reviewed and resolved. Data collected were the number of patients, preoperative patient characteristics, preoperative medications, surgery specifications, type and route of intervention, incidence of AF or supraventricular tachyarrhythmia, LOS, stroke, and postoperative follow up.

\section{Statistical analysis}

The occurrence of AF and stroke were treated as dichotomous variables and LOS was treated as a continuous variable. For comparison of LOS, the weighted mean difference was calculated as the difference between the mean values of LOS in treatment and control groups. Probability values of $\mathrm{p}<0.05$ were considered significant.

Analysis was based on the intention to treat principle. Pooled effect estimates and heterogeneity between studies were analysed by the random effects model with the RevMan 4.1 statistical package (The Cochran Collaboration, Information Management System, www.cc-ims.net/RevMan).

\section{RESULTS}

From the literature search and reference review, 20 studies that satisfied the inclusion criteria were found..$^{16-33}$ The publication dates spanned from 1982 to $2003 .{ }^{23}{ }^{25}$ Patient enrolment in single studies ranged from 20 to 400 patients. $^{22}{ }^{33}$ The total number of randomised patients was 2490 .

\section{Patient characteristics}

Table 1 summarises patient characteristics. Eighteen studies included patients undergoing CABG surgery. ${ }^{16-22}{ }^{24-31}$ 33-35 Four studies included at least some patients undergoing valve surgery, ${ }^{16202635}$ but in each of these studies the majority of patients enrolled underwent CABG procedures (range 88$100 \%)$ and only a minority underwent valve procedures (range 3-16\%). One study included only patients undergoing valve surgery. ${ }^{23}$ One study did not specify surgical procedure. $^{32}$ Aortic cross clamp time ranged from 38-103 minutes $^{2131}$ in the control group and 35-91 2631 minutes in the treatment group. Most of the studies excluded patients with abnormal renal function. ${ }^{16} 18192124-27303133$ Some excluded patients with severely decreased left ventricular function ${ }^{1619212729}$ and thyroid $^{1929}$ or respiratory disorders. ${ }^{16} 1921252730$

Some studies also excluded patients taking $\beta$ blockers, digoxin, calcium channel blockers, or diuretics preoperatively. In those trials that did not exclude patients being treated with preoperative $\beta$ blockers, the proportion of patients being treated ranged from $26-85 \% .{ }^{28}{ }^{34}$ For digoxin, the proportion of patients undergoing preoperative treatment ranged from $2-22 \% .{ }^{20}{ }^{31}$ For calcium channel blockers, the proportion ranged from $15-89 \% .{ }^{16}{ }^{24}$ For diuretics, the proportion ranged from $8-25 \%^{24} 31$

\section{Detection and treatment}

The majority of the trials used continuous ECG monitoring to detect arrhythmia. ${ }^{16-22} 25-2729303334$ Some of the studies used Holter recordings. ${ }^{24}{ }^{31}$ One study used a combination of continuous and Holter techniques. ${ }^{28}$ For some, the method of detection was not specified. ${ }^{23} 3235$ The length of continuous ECG monitoring ranged from 24 to 120 hours. ${ }^{18222627}$ The follow up period for arrhythmia detection was usually confined to hospital stay; however, in some trials there was a one month control visit. ${ }^{21}$

Regimens of magnesium administration varied and are summarised in table 2 .

\section{Atrial fibrillation}

Figure 1 presents the effect of magnesium on postoperative AF. Magnesium reduced the percentage of patients with AF from $28 \%$ in the control group to $18 \%$ in the treatment group (odds ratio (OR) $0.54,95 \%$ confidence interval (CI) 0.38 to $0.75)$, with significant heterogeneity between trials $(\mathrm{p}<0.001)$.

\begin{tabular}{|c|c|c|c|c|c|c|}
\hline Study & $\begin{array}{l}\text { Number of } \\
\text { participants }\end{array}$ & $\begin{array}{l}\text { Mean age } \\
\text { (years) }\end{array}$ & Men & $\begin{array}{l}\text { Mean LVEF } \\
(\%)\end{array}$ & Previous MI & Blinding \\
\hline Bert & $60 / 63$ & $64 / 63$ & $83 \% / 89 \%$ & $49 / 48$ & NA & NS \\
\hline Caspi & $48 / 50$ & $62 / 60$ & $79 \% / 68 \%$ & $38 / 40$ & $50 \% / 54 \%$ & NS \\
\hline Colquhoun & $64 / 66$ & $59 / 57$ & $80 \% / 83 \%$ & NA & $53 \% / 45 \%$ & DB \\
\hline Dagdelen & $55 / 93$ & $61 / 63$ & $78 \% / 77 \%$ & NA & NA & DB \\
\hline England & $50 / 50$ & $62 / 60$ & $68 \% / 58 \%$ & $48 / 47$ & NA & DB \\
\hline Fanning & $50 / 49$ & $62 / 59$ & $78 \% / 71 \%$ & $49 / 50$ & $42 \% / 35 \%$ & DB \\
\hline Forlani & $50 / 54$ & $64 / 64$ & $88 \% / 85 \%$ & $55 / 52$ & $66 \% / 65 \%$ & NS \\
\hline Harris & $11 / 9$ & $62 / 58$ & NA & $50 / 55$ & NA & DB \\
\hline Holden & $37 / 33$ & $45 / 47$ & $32 \% / 39 \%$ & NA & NA & DB \\
\hline Jensen & $28 / 29$ & $61 / 61$ & $100 \% / 100 \%$ & NA & NA & DB \\
\hline Kaplan & $100 / 100$ & $60 / 58$ & $74 \% / 76 \%$ & NA & $58 \% / 62 \%$ & NS \\
\hline Karmy Jones & $54 / 46$ & $60 / 65$ & $70 \% / 61 \%$ & $48 / 47$ & $54 \% / 50 \%$ & DB \\
\hline Nurozler & $25 / 25$ & $54 / 56$ & $92 \% / 92 \%$ & $66 / 67$ & $28 \% / 32 \%$ & DB \\
\hline Parikka & $71 / 69$ & $54 / 57$ & $82 \% / 84 \%$ & $59 / 61$ & NA & NS \\
\hline Schwieger & $100 / 100$ & $61 / 61$ & $64 \% / 74 \%$ & NA & NA & NB \\
\hline Shakerinia & $25 / 25$ & $65 / 67$ & $68 \% / 64 \%$ & $65 / 67$ & $72 \% / 80 \%$ & NS \\
\hline Toraman & $100 / 100$ & $61 / 62$ & $83 \% / 78 \%$ & NA & $53 \% / 49 \%$ & NS \\
\hline Treggiari-Venzi & $51 / 47$ & $65 / 65$ & $84 \% / 89 \%$ & $57 / 62$ & $45 \% / 38 \%$ & DB \\
\hline Wollert & $58 / 45$ & NA & NA & NA & NA & NS \\
\hline Yeatman & $200 / 200$ & $63 / 64$ & $78 \% / 83 \%$ & NA & NA & $D B$ \\
\hline
\end{tabular}

Blinding, method of blinding used in the study; DB, double blind study; LVEF, left ventricular ejection fraction; MI myocardial infarction; NA, data not available; NB, study not blinded; NS, blinding characteristics of study not specified. 


\begin{tabular}{|c|c|c|c|c|c|}
\hline Study & Infusion & $\begin{array}{l}\text { Treatment } \\
\text { initiation }\end{array}$ & Route & $\begin{array}{l}\text { Treatment } \\
\text { duration (hours) }\end{array}$ & $\begin{array}{l}\text { Total amount } \\
\text { (mmol) }\end{array}$ \\
\hline Bert & Magnesium sulfate & Postop & iv & 96 & 49 \\
\hline Caspi & Magnesium sulfate & Periop & iv & 24 & 48 \\
\hline Colquhoun & Magnesium chloride & Postop & iv & 48 & 50 \\
\hline Dagdelen & Magnesium sulfate & Preop & iv & 144 & 37 \\
\hline England & Magnesium chloride & Periop & iv & Intraop & 8 \\
\hline Fanning & Magnesium sulfate & Postop & iv & 96 & 84 \\
\hline Forlani & Magnesium sulfate & Preop & iv & 144 & 37 \\
\hline Harris & Magnesium chloride & Periop & iv & Intraop & 16 \\
\hline Holden & Magnesium sulfate & Postop & im & 72 & 7 \\
\hline Jensen & Magnesium sulfate & Postop & iv & 96 & 110 \\
\hline Kaplan & Magnesium sulfate & Preop & iv & 120 & 61 \\
\hline Karmy Jones & Magnesium sulfate & Postop & iv & 24 & 58 \\
\hline Nurozler & Magnesium sulfate & Postop & iv & 120 & 100 \\
\hline Parikka & Magnesium sulfate & Postop & iv & 48 & 70 \\
\hline Schwieger & Magnesium sulfate & NA & iv & 72 & Supp \\
\hline Shakerinia & Magnesium sulfate & Periop & iv & Intraop & NA \\
\hline Toraman & Magnesium sulfate & Preop & iv & 148 & 36 \\
\hline Treggiari-Venzi & Magnesium sulfate & Postop & iv & 72 & 48 \\
\hline Wollert & Magnesium sulfate & Postop & iv & 10 & 12 \\
\hline Yeatman & Magnesium sulfate & Periop & iv & Intraop & 20 \\
\hline
\end{tabular}

\section{Dosage}

To assess whether this heterogeneity was related to the dose of magnesium administered, trials were subdivided into low $(<35 \mathrm{mmol})$, medium $(35-50 \mathrm{mmol})$, and high (>50 mmol) dosages of magnesium. In the five trials with low dose magnesium (693 patients), the percentage of patients with AF was reduced from $31 \%$ in the control group to $20 \%$ in the treatment group (OR $0.50,95 \%$ CI 0.29 to 0.87 ). In the seven trials with medium dose magnesium (901 patients), the percentage of patients with AF was reduced from $32 \%$ in the control group to $17 \%$ in the treatment group (OR $0.36,95 \%$ CI 0.16 to 0.82 ). In the six trials with high dose magnesium (646 patients), the percentage of patients with AF was reduced from $23 \%$ in the control group to $20 \%$ in the treatment group (OR $0.87,95 \%$ CI 0.59 to $1.29)$.

\section{Timing}

To assess possible differences related to timing of magnesium administration, trials were subdivided into those with preoperative, perioperative, and postoperative regimens. In the four trials with preoperative magnesium administration (652 patients), the percentage of patients with AF was reduced from $25 \%$ in the control group to $8 \%$ in the treatment group (OR $0.19,95 \%$ CI 0.05 to 0.76 ). In the five trials with perioperative magnesium administration (668 patients), the percentage of patients with AF was reduced from 31\% in the control group to $25 \%$ in the treatment group (OR

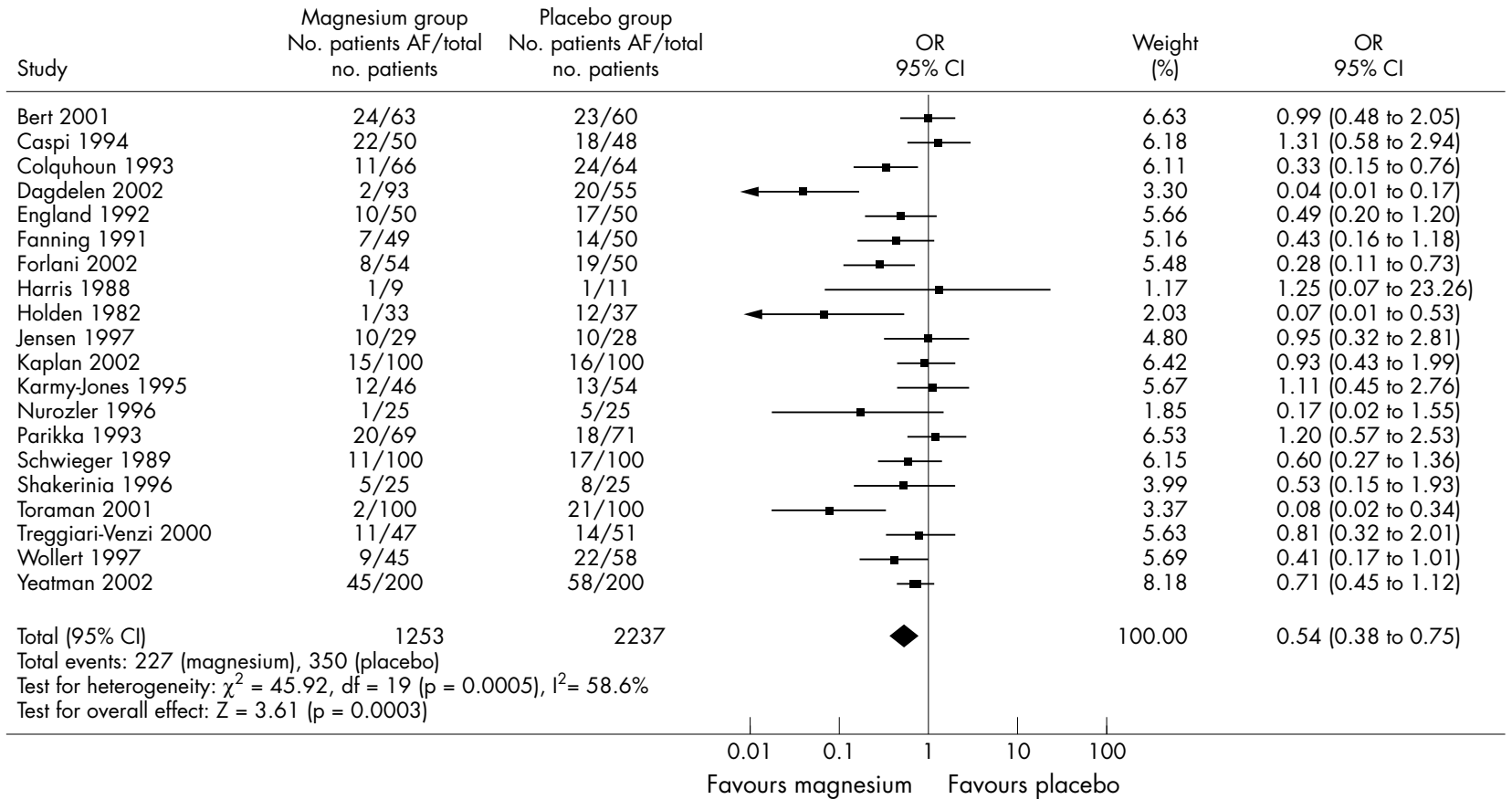

Figure 1 Incidence of postoperative atrial fibrillation. AF, atrial fibrillation developed postoperatively; $\mathrm{Cl}$, confidence interval; OR, odds ratio; total no. patients, total number of patients enrolled in the study. 


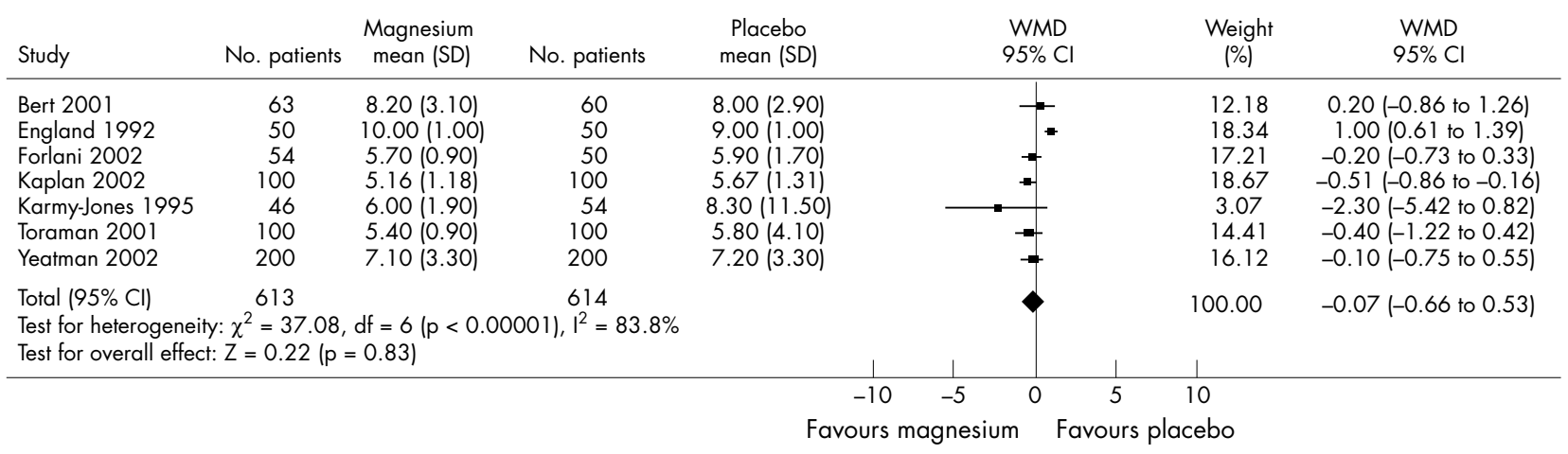

Figure 2 Effects of magnesium on hospital length of stay (days). WMD, weighted mean difference.

$0.74,95 \%$ CI 0.53 to 1.05$)$. In the 10 trials with postoperative magnesium administration (970 patients), the percentage of patients with AF was reduced from $31 \%$ in the control group to $22 \%$ in the treatment group (OR 0.63 , $95 \%$ CI 0.41 to 0.96 ).

\section{Surgery type}

To assess possible differences related to the type of surgery, trials were subdivided into those with patients undergoing CABG surgery only, CABG and valve surgery, and valve surgery only. In the 14 trials with CABG surgery only (1794 patients), the percentage of patients with AF was reduced from $28 \%$ in the control group to $17 \%$ in the treatment group (OR $0.51,95 \%$ CI 0.32 to 0.79 ). In the four trials with CABG and valve surgery ( 523 patients), the percentage of patients with $\mathrm{AF}$ was reduced from $26 \%$ in the control group to $22 \%$ in the treatment group (OR $0.77,95 \%$ CI 0.51 to 1.16 ). Only one study had valve surgery alone (70 patients), in which the percentage of patients with AF was reduced from 32\% to 3\% (OR $0.07,95 \%$ CI 0.01 to 0.53 ).

\section{LOS and mortality}

Seven trials reported the effect on LOS with a total of 1227 patients (fig 2). Magnesium did not significantly reduce LOS (weighted mean difference $-0.07,95 \%$ CI -0.66 to 0.53 ). No data were available on hospitalisation costs. Twelve trials reported data on postoperative mortality, with a total of 1471 patients. Overall mortality was $0.7 \%$ and was not significantly affected by magnesium (OR 1.22, 95\% CI 0.39 to 3.77 ).

\section{DISCUSSION \\ Main results}

This meta-analysis showed that magnesium administration significantly reduced the frequency of postoperative AF. Preoperative administration of magnesium was more effective in prevention of postoperative AF then intraoperative or postoperative prevention. Mortality and LOS were not affected significantly.

\section{Comparison with other prophylactic interventions}

A previous meta-analysis evaluated $\beta$ blockers, sotalol, amiodarone, and pacing as methods of prevention of postoperative $\mathrm{AF}^{36}$ All these interventions reduced postoperative AF significantly: $\beta$ blockers with OR of 0.39 (95\% CI 0.28 to $0.52)$; sotalol with OR of 0.35 (95\% CI 0.26 to 0.49 ); amiodarone with OR of 0.48 (95\% CI 0.37 to 0.61 ); and biatrial pacing with OR of 0.46 ( $95 \%$ CI 0.30 to 0.71 ). Our results suggest that magnesium is roughly as effective as most of the common prophylactic interventions in reducing postoperative $\mathrm{AF}$, with $\mathrm{OR}$ in the similar range.

\section{Adverse effects}

One problem with the use of antiarrhythmic agents to prevent postoperative AF is that the majority of patients do not develop postoperative AF after cardiac surgery but would still be exposed to possible side effects of prophylactic intervention. Routine prophylaxis of postoperative $\mathrm{AF}$ is estimated to expose $80 \%$ of patients to drugs for which there is no actual need. ${ }^{37}$ Intravenous magnesium is appealing because it avoids most of the proarrhythmic risks associated with other drugs. In general, few risks are associated with the prevention of hypomagnesaemia when renal function is normal..$^{38}$

\section{Mechanism of action}

The mechanisms by which magnesium administration reduces the incidence of postoperative AF are not entirely known. Depletion of magnesium, as shown in some studies of cardiac surgery patients, may be proarrhythmic. ${ }^{39-41}$ At the same time, administration of magnesium significantly increases atrial refractoriness. ${ }^{42}{ }^{43}$ The combination of the treatment effects of correcting magnesium depletion and of increasing atrial refractoriness caused by magnesium administration is probably responsible for the overall beneficial effect of magnesium.

\section{Heterogeneity}

There was significant heterogeneity among the trials, attributable to a number of factors such as different exclusionary criteria, various arrhythmia monitoring techniques, diverse definitions of $\mathrm{AF}$, the surgical procedure performed, and alternative administration regimens and dosages of magnesium. Subdivision of the trials by times of initiation showed that preoperative administration was most effective in reducing postoperative AF. Hypomagnesaemia can occur before cardiac surgery in some patients ${ }^{10}{ }^{12}$ and so this provides a possible explanation for the increased effectiveness of preoperative administration. Subdivision of the trials by the total amount of magnesium administered showed that a low dose of magnesium was most effective in reducing postoperative AF. Other individual studies have shown that a high dose of magnesium was significantly more effective then a low dose in preventing $\mathrm{AF}^{44}$ Subdivision of the trials by surgery type showed that magnesium was more effective in trials with CABG surgery alone than when CABG was combined with valve surgery. Magnesium appeared to be most effective in the single trial in which only valve operations were performed. Factors such as length of treatment may have confounded the analyses, so the above conclusions should be treated cautiously. 


\section{LOS and mortality}

Postoperative AF has been associated with LOS extended by three to four days ${ }^{7}$ and accompanying increases in hospital costs. ${ }^{44}{ }^{45}$ Magnesium did not significantly reduce the LOS in this meta-analysis. Fewer than half of patients will develop postoperative AF and still fewer develop prolonged $A F$, so the effect of magnesium on LOS in patients prone to AF would have to be very large to be able to detect an effect of LOS in the total population. It is possible that postoperative AF is a confounding variable and not necessarily the cause of the associated adverse events. Since only seven studies provided data on LOS, additional studies or data are warranted. Likewise, because mortality rates after cardiac surgery are very low, no trial of sufficient statistical power can realistically be done. Only two trials reported data on postoperative stroke. ${ }^{30}{ }^{33}$ Therefore, the effect of magnesium on postoperative stroke could not be determined.

\section{Limitations}

This meta-analysis is limited by the lack of complete availability of relevant data, particularly for LOS, stroke, and mortality. The studies we analysed span over 20 years during which time there was an intensive evolution of cardiac surgery techniques. Nevertheless, treatment benefit seemed to be similar over time. Some studies excluded groups of high risk but commonly encountered cardiac patients. This limits the extrapolation of the data from the clinical trials into everyday practice. Lastly, even though an examination search was done, meta-analysis itself is associated with a publication bias in that studies with a positive result are more likely to be published than negative studies.

\section{Conclusion}

Magnesium administration is effective for reducing postoperative AF, with a magnitude of the effect comparable with that of proposed antiarrhythmic drugs. Magnesium administration did not significantly reduce hospital LOS or mortality. Questions remain about the optimal regimen of administration and its efficacy in combination with other drugs.

\section{ACKNOWLEDGEMENT}

The summer student grant came from the Department of Medicine, Sunnybrook and Women's College Health Sciences Centre.

\section{Authors' affiliations \\ S Miller, E Crystal, M Garfinkle, C Lau, I Lashevsky, Arrhythmia} Services, Schulich Heart Centre, Department of Medicine, Faculty of Medicine, University of Toronto, Toronto, Ontario, Canada S J Connolly, Department of Medicine, McMaster University, Hamilton, Ontario, Canada

\section{REFERENCES}

1 Fuller JA, Adams GG, Buxton B. Atrial fibrillation after coronary artery bypass grafting: is it a disorder of the elderly? J Thorac Cardiovasc Surg 1989;97:821-5.

2 Mathew JP, Parks R, Savino JS, et al. Atrial fibrillation following coronary artery bypass graft surgery: predictors, outcomes, and resource utilization. Multicenter study of perioperative ischemia research group. JAMA 1996;276:300-6.

3 Ommen SR, Odell JA, Stanton MS. Atrial arrhythmias after cardiothoracic surgery. N Engl J Med 1997;336:1429-34.

4 Hogue CW Jr, Hyder ML. Atrial fibrillation after cardiac operation: risks, mechanisms, and treatment. Ann Thorac Surg 2000;69:300-6.

5 Olshansky B. Management of atrial fibrillation after coronary artery bypass graft. Am J Cardiol 1996;78:27-34.

6 Almassi GH, Schowalter T, Nicolosi AC, et al. Atrial fibrillation after cardiac surgery: a major morbid event? Ann Surg 1997;226:501-11; discussion
7 Creswell LL, Schuessler RB, Rosenbloom M, et al. Hazards of postoperative atrial arrhythmias. Ann Thorac Surg 1993;56:539-49.

8 Leitch JW, Thomson D, Baird DK, et al. The importance of age as a predictor of atrial fibrillation and flutter after coronary artery bypass grafting. J Thorac Cardiovasc Surg 1990;100:338-42.

9 Holden MP, lonescu Ml, Wooler GH. Magnesium in patients undergoing open-heart surgery. Thorax 1972;27:212-8.

10 Aglio LS, Stanford GG, Maddi R, et al. Hypomagnesemia is common following cardiac surgery. J Cardiothorac Vasc Anesth 1991;5:201-8.

11 Khan RM, Hodge JS, Bassett HF. Magnesium in open-heart surgery. J Thorac Cardiovasc Surg 1973;66:185-91.

12 Satur CM. Magnesium and cardiac surgery. Ann R Coll Surg Engl 1997;79:349-54.

13 Vyvyan HA, Mayne PN, Cutfield GR. Magnesium flux and cardiac surgery: a study of the relationship between magnesium exchange, serum magnesium levels and postoperative arrhythmias. Anaesthesia 1994;49:245-9.

14 Casthely PA, Yoganathan T, Komer C, et al. Magnesium and arrhythmias after coronary artery bypass surgery. J Cardiothorac Vasc Anesth 1994:8:188-91.

15 Speziale G, Ruvolo G, Fattouch K, et al. Arrhythmia prophylaxis after coronary artery bypass grafting: regimens of magnesium sulfate administration. Thorac Cardiovasc Surg 2000;48:22-6.

16 Bert AA, Reinert SE, Singh AK. A beta-blocker, not magnesium, is effective prophylaxis for atrial tachyarrhythmias after coronary artery bypass graft surgery. J Cardiothorac Vasc Anesth 2001;15:204-9.

17 Caspi J, Rudis E, Bar I, et al. Effects of magnesium on myocardial function after coronary artery bypass grafting. Ann Thorac Surg 1995;59:942-7.

18 Colquhoun IW, Berg GA, el-Fiky M, et al. Arrhythmia prophylaxis after coronary artery surgery: a randomised controlled trial of intravenous magnesium chloride. Eur J Cardiothorac Surg 1993;7:520-3.

19 Dagdelen S, Toraman F, Karabulut H, et al. The value of P dispersion on predicting atrial fibrillation after coronary artery bypass surgery: effect of magnesium on P dispersion. Ann Noninvasive Electrocardiol 2002;7:21 1-8.

20 England MR, Gordon G, Salem M, et al. Magnesium administration and dysrhythmias after cardiac surgery: a placebo-controlled, double-blind, randomized trial. JAMA 1992;268:2395-402.

21 Forlani S, De Paulis R, de Notaris S, et al. Combination of sotalol and magnesium prevents atrial fibrillation after coronary artery bypass grafting. Ann Thorac Surg 1992;268:720-5; discussion 725-6.

22 Harris MN, Crowther A, Jupp RA, et al. Magnesium and coronary revascularization. Br J Anaesth 1988;60:779-83.

23 Holden MP. Value of magnesium supplements during open heart surgery: a double blind trial. In: HK N, ed. Nutrition and heart disease. Jamaica, New Yoek: SP Medical and Scientific Books, 1982:273-83.

24 Jensen BM, Alstrup P, Klitgard NA. Magnesium substitution and postoperative arrhythmias in patients undergoing coronary artery bypass grafting. Scand Cardiovasc J 1997;31:265-9.

25 Kaplan M, Kut MS, Icer UA, et al. Intravenous magnesium sulfate prophylaxis for atrial fibrillation after coronary artery bypass surgery. J Thorac Cardiovasc Surg 2003; 125:344-52

26 Karmy-Jones R, Hamilton A, Dzavik V, et al. Magnesium sulfate prophylaxis after cardiac operations. Ann Thorac Surg 1995;59:502-7.

27 Nurozler F, Tokgozoglu L, Pasaoglu I, et al. Atrial fibrillation after coronary artery bypass surgery: predictors and the role of $\mathrm{MgSO} 4$ replacement. J Card Surg 1996;11:421-7.

28 Parikka H, Toivonen L, Pellinen T, et al. The influence of intravenous magnesium sulphate on the occurrence of atrial fibrillation after coronary artery by-pass operation. Eur Heart J 1993;14:251-8.

29 Shakerinia T, Ali IM, Sullivan JA. Magnesium in cardioplegia: Is it necessary? Can J Surg 1996;1996:397-400.

30 Toraman F, Karabulut EH, Alhan $\mathrm{HC}$, et al. Magnesium infusion dramatically decreases the incidence of atrial fibrillation after coronary artery bypass grafting. Ann Thorac Surg 2001;72:1256-61; discussion 1261-2.

31 Treggiari-Venzi MM, Waeber JL, Perneger TV, et al. Intravenous amiodarone or magnesium sulphate is not cost-beneficial prophylaxis for atrial fibrillation after coronary artery bypass surgery. Br J Anaesth 2000;85:690-5.

32 Wollert HG, Grossmann H, Eckel L. Magnesium sulfate prevents atrial tachyarrhythmias after cardiac and non-cardiac surgery: what is the common mechanism? Thorac Cardiovasc Surg 1997:45:213-4.

33 Yeatman M, Caputo M, Narayan P, et al. Magnesium-supplemented warm blood cardioplegia in patients undergoing coronary artery revascularization. Ann Thorac Surg 2002;73:112-8.

34 Fanning WJ, Thomas CS Jr, Roach A, et al. Prophylaxis of atrial fibrillation with magnesium sulfate after coronary artery bypass grafting. Ann Thorac Surg 1991;52:529-33.

35 Schwieger IM, Kopel ME, Finlayson DC. Magnesium and postoperative dysrhythmias in patients after cardiac surgery. J Cardiothorac Anesth 1989:3:18.

36 Crystal E, Connolly SJ, Sleik K, et al. Interventions on prevention of postoperative atrial fibrillation in patients undergoing heart surgery: a metaanalysis. Circulation 2002;106:75-80.

37 Hogue CW Jr. Con: All patients should not receive pharmacologic prophylaxis for atrial fibrillation after cardiac surgery. J Cardiothorac Vasc Anesth 1999;13:101-4.

38 Hilleman D, Miller MA, Parker R, et al. Optimal management of amiodarone therapy: efficacy and side effects. Pharmacotherapy 1998; 18:138S-45S

39 Klevay LM, Milne DB. Low dietary magnesium increases supraventricular ectopy. Am J Clin Nutr 2002;75:550-4.

40 Guideri G, Lehr D, Horowitz S. Enhanced incidence of isoproterenol-induced ventricular fibrillation in the magnesium-deficient rat. J Am Coll Nutr 1985;4:139-55. 
41 Roden DM, lansmith DH. Effects of low potassium or magnesium concentrations on isolated cardiac tissue. Am J Med 1987;82:18-23.

42 Rasmussen HS, Thomsen PE. The electrophysiological effects of intravenous magnesium on human sinus node, atrioventricular node, atrium, and ventricle. Clin Cardiol 1989;12:85-90.

43 Christiansen EH, Frost L, Andreasen F, et al. Dose-related cardiac electrophysiological effects of intravenous magnesium: a double-blind placebo-controlled dose-response study in patients with paroxysmal supraventricular tachycardia. Europace 2000;2:320-6.

44 Wistbacka JO, Koistinen J, Karlqvist KE, et al. Magnesium substitution in elective coronary artery surgery: a double-blind clinical study. J Cardiothorac Vasc Anesth 1995:9:140-6.

45 Aranki SF, Shaw DP, Adams DH, et al. Predictors of atrial fibrillation after coronary artery surgery: current trends and impact on hospital resources. Circulation 1996;94:390-7.

\section{IMAGES IN CARDIOLOGY}

doi: 10.1136/hrt.2004.042598

Apical left ventricular aneurysm presenting with malignant ventricular tachycardia responsive to aneurysmectomy

A

76 year old man with no prior cardiac history was admitted after three episodes of syncope. With symptoms, telemetry revealed a rapid monomorphic rhythm (panel A) consistent with ventricular tachycardia (VT). ECG and cardiac markers were unremarkable. Echocardiography revealed an apical defect emptying into a large thin-walled chamber (panels B, C, D). Doppler interrogation revealed bidirectional flow between the left ventricle (LV) and this chamber, consistent with true aneurysm of the LV apex versus contained rupture of the LV apex with pseudoaneurysm formation. Cardiac catheterisation showed a $40 \%$ plaque in the mid left anterior descending coronary artery. At cardiac surgery, the abnormality was resected and the LV repaired with a pericardial patch. Recovery was uneventful with no further VT on telemetry and no VT inducible at electrophysiology study. Pathological examination of the resected tissue revealed true aneurysm with surviving myocytes scattered throughout areas of scar. Aetiology was likely an old myocardial

infarction, caused by left anterior descending coronary artery thrombosis or spasm at the site of a focal and intrinsically non-obstructive plaque.

Differentiation of true aneurysm from pseudoaneurysm by echocardiography can be difficult. Here, true aneurysm was indicated by the presence of continuous endomyocardial and pericardial layers extending from the normal portion of the left ventricle throughout the aneurysmal sac. Aneurysms of the left ventricle, often associated with ventricular arrhythmias, typically form by dilation of infarct or infarct scars, although congenital and inflammatory aneurysms have been described. Resection of the aneurysm and surrounding abnormal myocardium can cure the ventricular arrhythmias, as in this case.

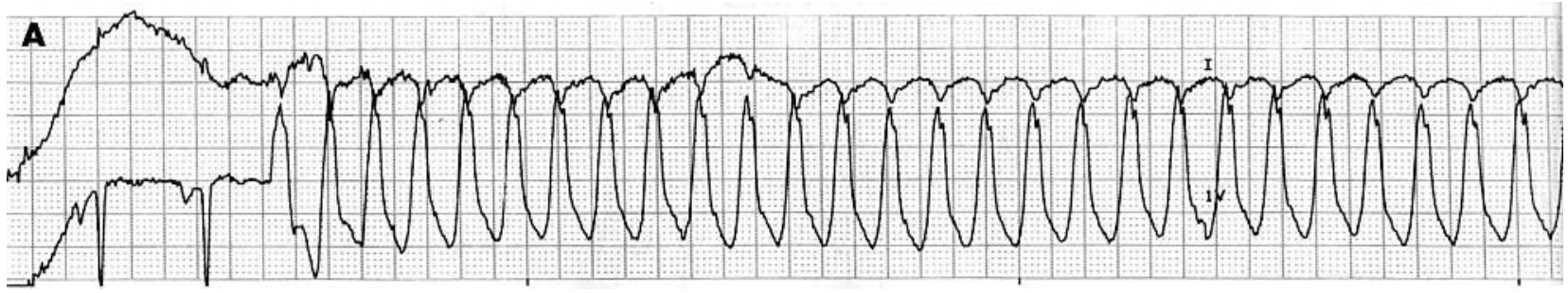

Telemetry strip of leads I and V1 demonstrating the spontaneous onset of monomorphic ventricular tachycardia with a right bundle branch block pattern at approximately 280 beats $/ \mathrm{min}$.
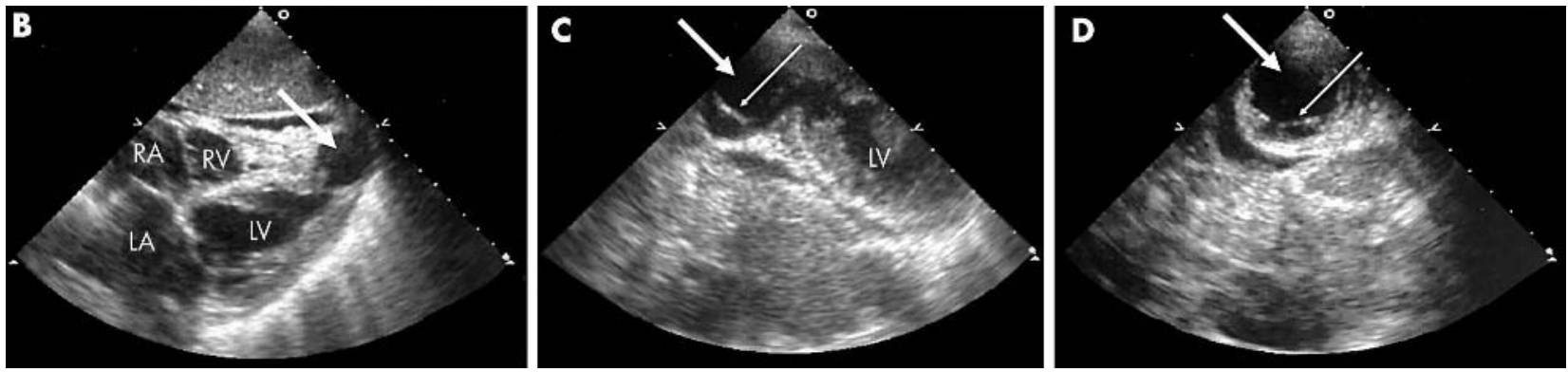

Subcostal (B) and off-axis apical (C) views from transthoracic echocardiogram demonstrating left ventricular apical defect. Panel D is another apical view showing a cross section of the aneurysmal sac. Heavy arrows indicate an echo-free chamber communicating with the left ventricular cavity by an orifice with a narrow neck. Here, the definitive feature distinguishing true aneurysm from pseudoaneurysm is the presence of an endomyocardial border lining the cavity, as well as a continuous pericardial layer. Thin arrows indicate the residual myocardial layer in the wall of the aneurysmal chamber. Other findings that have been described to help distinguish between these two entities are the ratio of the maximal diameter of the neck to maximal diameter of the cavity ( 0.25 and 0.5 for pseudoaneurysm and 0.9 to 1 for aneurysm), and the flow characteristics of the chamber (turbulent flow by pulse Doppler in neck or within cavity in the pseudoaneurysm), which were not helpful in this case. LA, left atrium; LV, left ventricle; RA, right atrium; RV, right ventricle. 